Article

\title{
The Acute Effects of Caffeinated Black Coffee on Cognition and Mood in Healthy Young and Older Adults
}

\author{
Crystal F. Haskell-Ramsay ${ }^{1, *} \mathbb{C}^{\mathbb{C}}$, Philippa A. Jackson ${ }^{1}{ }^{\mathbb{B}}$, Joanne S. Forster ${ }^{1}$, Fiona L. Dodd ${ }^{1}$, \\ Samantha L. Bowerbank ${ }^{2}$ (i) and David O. Kennedy ${ }^{1}$ \\ 1 Brain, Performance and Nutrition Research Centre, Northumbria University, Newcastle Upon-Tyne NE1 8ST, \\ UK; philippa.jackson@northumbria.ac.uk (P.A.J.); jo.forster@northumbria.ac.uk (J.S.F.); \\ f.dodd@northumbria.ac.uk (F.L.D.); david.kennedy@northumbria.ac.uk (D.O.K.) \\ 2 Faculty of Health and Life Sciences, Northumbria University, Newcastle Upon-Tyne NE1 8ST, UK; \\ samantha.bowerbank@northumbria.ac.uk \\ * Correspondence: crystal.haskell-ramsay@northumbria.ac.uk; Tel.: +44-191-2274875
}

Received: 20 August 2018; Accepted: 24 September 2018; Published: 30 September 2018

check for updates

\begin{abstract}
Cognitive and mood benefits of coffee are often attributed to caffeine. However, emerging evidence indicates behavioural effects of non-caffeine components within coffee, suggesting the potential for direct or synergistic effects of these compounds when consumed with caffeine in regular brewed coffee. The current randomised, placebo-controlled, double-blind, counterbalanced-crossover study compared the effects of regular coffee, decaffeinated coffee, and placebo on measures of cognition and mood. Age and sex effects were explored by comparing responses of older (61-80 years, $N=30$ ) and young (20-34 years, $N=29$ ) males and females. Computerised measures of episodic memory, working memory, attention, and subjective state were completed at baseline and $30 \mathrm{~min}$ post-drink. Regular coffee produced the expected effects of decreased reaction time and increased alertness when compared to placebo. When compared to decaffeinated coffee, increased digit vigilance accuracy and decreased tiredness and headache ratings were observed. Decaffeinated coffee also increased alertness when compared to placebo. Higher jittery ratings following regular coffee in young females and older males represented the only interaction of sex and age with treatment. These findings suggest behavioural activity of coffee beyond its caffeine content, raising issues with the use of decaffeinated coffee as a placebo and highlighting the need for further research into its psychoactive effects.
\end{abstract}

Keywords: coffee; caffeine; chlorogenic acids; phenolic; cognition; cognitive; mood; age; sex

\section{Introduction}

Coffee consumption is associated with a number of health benefits in elderly men and women including reduced risk of cardiovascular disease (CVD) [1], lower incidence of type 2 diabetes mellitus [2], and decreased death from inflammatory diseases [3], CVD [4,5], and all-cause mortality [6,7]. A number of physiological factors associated with these conditions are relevant to cognitive function in healthy ageing, as well as pathological ageing conditions such as dementia or Alzheimer's disease (AD). Indeed, a number of epidemiological studies have demonstrated an association between higher coffee consumption and better performance on cognitive tests in older adults [8,9], as well as an inverse relationship between coffee consumption and risk of dementia/AD [10-14]. 
Cognitive benefits from coffee consumption are typically attributed to caffeine, which exerts its effects through non-selective antagonism of adenosine $\mathrm{A}_{1}$ and $\mathrm{A}_{2 \mathrm{~A}}$ receptors [15]. In support of this, a number of studies have demonstrated the ability of caffeine to improve measures of attention and increase ratings of alertness [16-18]. However, coffee contains more than 1000 different compounds including phenolics, diterpenes, and melanoidins [19], all of which have the potential to affect behaviour either directly or indirectly through interaction with caffeine. This is demonstrated by studies showing direct psychoactive effects and modulation of caffeine's effects by the amino acid 1-theanine, present in tea [20-22]. Similarly, lengthened startled blink onset latency has been shown following decaffeinated coffee as compared to caffeinated coffee, caffeinated juice, and non-caffeinated juice [23]. Chlorogenic acids (CGA) are a group of phenolic compounds representing the principal non-caffeine components in coffee [24] and have been explored in relation to mood and cognition in healthy, elderly participants [25]. In comparison to regular CGA decaffeinated coffee (224 mg CGA, $5 \mathrm{mg}$ caffeine), high CGA decaffeinated coffee (521 mg CGA, $11 \mathrm{mg}$ caffeine) increased alertness and decreased negative emotional processing, whereas caffeinated coffee (244 mg CGA, $167 \mathrm{mg}$ caffeine) increased accuracy on a sustained attention task and improved mood. These results indicate that the addition of CGA to regular decaffeinated coffee can modulate its effects on behaviour. The effects of CGA were explored further in a study comparing $540 \mathrm{mg}$ isolated CGA, $6 \mathrm{~g}$ decaffeinated green blend coffee (532 mg CGA), and placebo [26]. Whilst positive effects on mood were observed following decaffeinated green blend coffee, these effects were not evident following CGA in isolation, which also led to detrimental effects to cognition at $120 \mathrm{~min}$ post-drink. This provides further evidence for behavioural effects of decaffeinated coffee and highlights the need to consider the synergistic contribution of non-caffeine compounds in coffee.

Coffee is one of the most widely consumed beverages in the world, yet intervention trials examining the specific impact of consuming regular, brewed coffee on cognition and mood are lacking. Given the potential for non-caffeine components within coffee to exert psychoactive effects or to interact synergistically with caffeine, it is important that the effects of regular coffee and decaffeinated coffee are compared to placebo. In addition, despite physiological differences between men and women, including in their nutrient needs and in cognitive performance $[27,28]$, sex differences are rarely considered in nutritional intervention trials. This is particularly important here, as studies of the relationship between coffee and cognitive decline have indicated that whilst reduced risk is related to coffee consumption in men [29], the effect is more pronounced in women [30,31]. This suggests that greater effects of coffee consumption may be observed in older adults as a consequence of cognitive decline, and that these beneficial effects may be enhanced in females. Furthermore, given the impact of the menstrual cycle on resting metabolic rate [32] and systemic clearance of caffeine [33], it is also possible that sex differences in response will be moderated by age. In order to explore this further, the current study compared the behavioural effects of regular coffee, decaffeinated coffee and placebo in elderly participants (61-80 years) to those in a younger (20-34 years) adult group and examined differential responses in men and women. As debate continues as to whether caffeine's effects are modulated by habitual consumption $[34,35]$ only those who regularly consumed coffee and tea were included.

\section{Materials and Methods}

\subsection{Design}

A randomised, placebo-controlled, double-blind, counterbalanced-crossover design was employed. The study was approved by Northumbria University's Faculty of Health and Life Sciences Ethics Committee (reference: SUB057_Forster_090216; approved: 26 February 2016) and was conducted in accordance with the Declaration of Helsinki. 


\subsection{Participants}

Seventy-two participants were drawn through an opportunity sample within Newcastle upon Tyne and the surrounding areas. Thirty-six of these represented an older group aged 61 to 80 years (18 male), and 36 represented a younger comparator group aged 20 to 34 years (18 male). Sample size was determined from a power calculation based upon previous data showing improvements to cognition and mood in habitual caffeine consumers following $150 \mathrm{mg}$ caffeine [16]. An effect size of $d=0.6$ indicated that a total of 72 participants would allow detection of significant effects with a power of 0.8. All participants were healthy non-smokers for whom English was their first language. Participants were not currently taking medication with the exception of contraception in young female participants and those used in the treatment of arthritis, high blood pressure, high cholesterol, and reflux-related conditions in the older participant group. Due to the potential impact of habitual caffeine intake on response, only those who regularly consumed more than two cups of coffee or three cups of tea (equating to $\geq 150 \mathrm{mg}$ caffeine/day) were included. Participants were paid $£ 60$ for taking part.

Thirteen participants were excluded from the per protocol analysis (12 based on high $(>1 \mu \mathrm{g} / \mathrm{mL}$ ) pre-dose caffeine salivary levels, and one due to under-consumption of the drink provided). The population for analysis (see Figure 1) consisted of 30 older adults (14 male) and 29 young (16 males). Participant characteristics for each age group by sex can be found in Table 1.

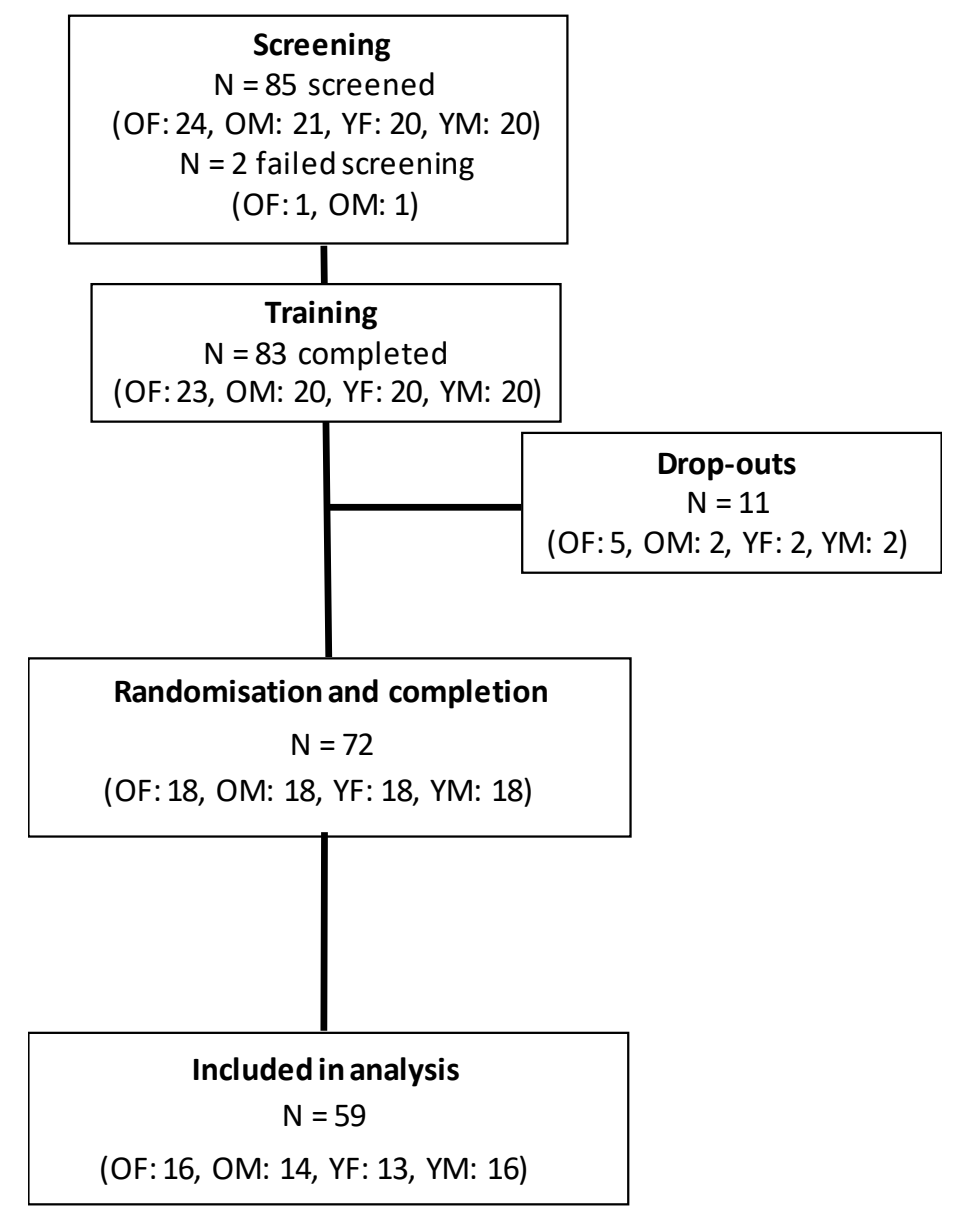

Figure 1. Final participant disposition. $\mathrm{N}=$ Number of participants; $\mathrm{OF}=$ Older Female; $\mathrm{OM}=$ Older Male; YF = Younger Female; YM = Younger Male. 
Table 1. Participant demographics (SD = Standard Deviation).

\begin{tabular}{|c|c|c|c|c|c|c|c|c|}
\hline & \multicolumn{4}{|c|}{ Young } & \multicolumn{4}{|c|}{ Older } \\
\hline & \multicolumn{2}{|c|}{ Male } & \multicolumn{2}{|c|}{ Female } & \multicolumn{2}{|c|}{ Male } & \multicolumn{2}{|c|}{ Female } \\
\hline & Mean & SD & Mean & SD & Mean & SD & Mean & SD \\
\hline Age & 26.3 & 4.4 & 26.2 & 3.6 & 67.7 & 6.3 & 67.1 & 3.4 \\
\hline Years in education & 18 & 3 & 17 & 3 & 16 & 5 & 14 & 4 \\
\hline Body Mass Index (BMI) & 25.7 & 3.8 & 23.8 & 3.6 & 25.9 & 3.4 & 26.1 & 3.9 \\
\hline Caffeine consumption (mg/day) & 327 & 88.2 & 351 & 110.4 & 426 & 74.4 & 394 & 87.8 \\
\hline Coffee consumption (cups/day) & 2.88 & 1.54 & 2.54 & 1.45 & 2.64 & 1.13 & 3.59 & 0.93 \\
\hline Fruit and vegetables (portions/day) & 4.3 & 1.5 & 4.1 & 1.4 & 4.4 & 1.9 & 5.4 & 1.8 \\
\hline
\end{tabular}

\subsection{Treatment}

At each study visit, one of the following drinks was administered by an independent third party with no further involvement in the study.

- $\quad 220 \mathrm{~mL}$ water mixed with $2.5 \mathrm{~g}$ coffee flavouring (placebo)

- $220 \mathrm{~mL}$ regular coffee (without milk and sugar) containing $100 \mathrm{mg}$ caffeine

- $220 \mathrm{~mL}$ decaffeinated coffee (without milk and sugar) containing $\sim 5 \mathrm{mg}$ caffeine

The order in which participants received each drink was determined by computer-generated random allocation (Latin square) for each sex (male, female) by group (older and younger age comparators). Regular and decaffeinated coffee were brewed using two separate drip filter coffee makers following a standardised brewing procedure, including the use of filter papers to minimise cafestol and kahweol levels. Placebo consisted of $2.5 \mathrm{~g}$ flavouring (maltodextrin $2.26 \mathrm{~g}$, dark roast $0.1 \mathrm{~g}$, mild roast $0.1 \mathrm{~g}$, and coffee natural $0.04 \mathrm{~g}$-Firmenich SA, Meyrin, Satigny, Switzerland) added to boiling water. Drinks were matched for temperature $\left(58^{\circ} \mathrm{C}\right)$ and served in an opaque thermal beaker with a black opaque straw with 5 min allowed for drinking.

\subsection{Salivary Caffeine Levels}

Saliva samples were obtained using salivettes (Sarstedt, Leicester, UK). Samples were taken immediately prior to baseline assessments in order to confirm compliance to abstinence and following post-drink assessments to confirm effective caffeine absorption. The saliva samples were immediately frozen at $-20{ }^{\circ} \mathrm{C}$ until thawing. Once thawed, salivette tubes were centrifuged at $15,000 \times g$ for $10 \mathrm{~min}$. Stock solutions of caffeine, paraxanthine, and benzotriazole (internal standard) were prepared in type I ultra-pure water at a concentration of $100 \mu \mathrm{g} / \mathrm{mL}$. Calibration standards for caffeine and paraxanthine were prepared between 0.05 and $5.00 \mu \mathrm{g} / \mathrm{mL}$. Quality control samples were also prepared at a concentration of $2.5 \mu \mathrm{g} / \mathrm{mL}$. Internal standard $(50 \mu \mathrm{L}$ at $5.0 \mu \mathrm{g} / \mathrm{mL})$ was added to $50 \mu \mathrm{L}$ of each standard and sample in duplicate. To extract the compounds $2 \mathrm{~mL}$ of ethyl acetate was added and solutions were vortex mixed for $3 \mathrm{~min}$ following by centrifugation at $4000 \times \mathrm{g}$ for $10 \mathrm{~min}$. The organic layer was transferred to a clean tube and dried under a stream of nitrogen at $45^{\circ} \mathrm{C}$. The residue was reconstituted in $100 \mu \mathrm{L}$ of mobile phase and $50 \mu \mathrm{L}$ injected onto the column.

Saliva samples were analysed with high-performance liquid chromatography. The HPLC system was an Agilent 1260 Infinity ${ }^{\mathrm{TM}}$ (Cheadle, Greater Manchester, UK) consisting of an Infinity ${ }^{\mathrm{TM}}$ quaternary pump, an Infinity ${ }^{\mathrm{TM}}$ Autosampler with integrated column oven and an Infinity ${ }^{\mathrm{TM}}$ multi-wavelength detector set at $280 \mathrm{~nm}$. Instrument control and data processing was performed using Agilent OpenLab ${ }^{\mathrm{TM}}$ CDS (Agilent Technologies Ltd., Cheadle, UK). Chromatographic separation was achieved on a Kinetex $\mathrm{C}_{18}$ column $(4.6 \times 250 \mathrm{~mm}$ i.d., particle size $5 \mu \mathrm{m}$; Phenomenex Ltd., Macclesfield, UK). The mobile phase consisted of acetonitrile, acetic acid and type I ultra-pure water (5:1:95, $v \%: v \%: v \%)$ delivered at a flow rate of $1.00 \mathrm{~mL} / \mathrm{min}$. 


\subsection{Cognitive and Mood Measures}

With the exception of driving ability, all cognitive and mood measures were delivered using the Computerised Mental Performance Assessment System (COMPASS, Northumbria University, Newcastle upon Tyne, UK), a purpose-designed software application for the flexible delivery of randomly generated parallel versions of standard and novel cognitive assessment tasks. This assessment system has previously been shown to be sensitive to nutritional interventions $[36,37]$ including caffeine [20]. The tasks and mood scales were chosen based on their known sensitivity to caffeine or susceptibility to ageing. Tasks were presented in the same order on each occasion and, with the exception of the paper and pencil tasks (immediate and delayed word recall and verbal fluency), responses were made using a response pad. The entire selection of tasks took approximately $25 \mathrm{~min}$ to complete. See Table 2 for order and scoring of tasks completed at baseline. Due to the potential for interference from repeat completions, computerised location learning and driving simulation were only completed post-dose on study visits with the final session from their training day used as the statistical baseline in the analyses.

Table 2. Cognitive tasks completed at baseline and $30 \mathrm{~min}$ post-dose in order of presentation (computerised location learning and driving ability are described below).

\begin{tabular}{|c|c|c|c|}
\hline Task & Descriptor & Scoring & Domain \\
\hline Word presentation & $\begin{array}{l}\text { A series of words is displayed on the screen, } \\
\text { one word at a time. In this case, } 15 \text { words were } \\
\text { presented with a display time of } 1 \mathrm{~s} \text { and } \\
\text { interstimulus interval of } 1 \mathrm{~s}\end{array}$ & - & \\
\hline Immediate word recall & $\begin{array}{l}\text { Participants are instructed to write down the } \\
\text { words that were presented. In this case, } 60 \mathrm{~s} \\
\text { were given to complete the task }\end{array}$ & $\begin{array}{l}\text { Number correct and } \\
\text { number of errors }\end{array}$ & Episodic memory \\
\hline Picture presentation & $\begin{array}{l}\text { A series of photographic images are displayed } \\
\text { on the screen, one at a time. In this case, } 15 \\
\text { images were presented with a display time of } 2 \\
\mathrm{~s} \text { and an interstimulus interval of } 1 \mathrm{~s}\end{array}$ & - & \\
\hline Simple reaction time & $\begin{array}{l}\text { An upwards pointing arrow is displayed on the } \\
\text { screen at irregular intervals. Participants must } \\
\text { respond as quickly as they can as soon as they } \\
\text { see the arrow appear. In this case, } 50 \text { stimuli } \\
\text { were presented }\end{array}$ & Reaction time (ms) & Attention \\
\hline Digit vigilance & $\begin{array}{l}\text { A fixed number appears on the right of the } \\
\text { screen and a series of changing numbers } \\
\text { appear on the left of the screen at the rate of } \\
150 \text { per minute. Participants are required to } \\
\text { make a response when the number on the left } \\
\text { matches the number on the right. In this case } \\
\text { the task lasted for } 3 \text { min }\end{array}$ & $\begin{array}{l}\text { Accuracy }(\%) \text {, reaction } \\
\text { time for the correct } \\
\text { responses (ms) and false } \\
\text { alarms (number) }\end{array}$ & Attention \\
\hline $\begin{array}{l}\text { Numeric working } \\
\text { memory }\end{array}$ & $\begin{array}{l}\text { Five single target numbers are displayed on the } \\
\text { screen, one at a time. Participants are required } \\
\text { to memorise these numbers as they appear. } \\
\text { Once the target series has been presented, } \\
\text { numbers are displayed one at a time and } \\
\text { participants are required to indicate if each } \\
\text { number was presented in the previous list or } \\
\text { not. In this case, three trials were completed }\end{array}$ & $\begin{array}{l}\text { Accuracy }(\%) \text { and } \\
\text { reaction time for the } \\
\text { correct responses (ms) }\end{array}$ & Working memory \\
\hline Verbal fluency & $\begin{array}{l}\text { Participants are presented with a letter on } \\
\text { screen and asked to write down as many words } \\
\text { as they can, beginning with that letter. In this } \\
\text { case, the letters presented were A, T, C, F, M, } \\
\text { and S and } 60 \text { s were given to complete the task }\end{array}$ & $\begin{array}{l}\text { Number correct } \\
\text { permitted words, with } \\
\text { names and } \\
\text { perseverations } \\
\text { discounted from the total } \\
\text { score }\end{array}$ & Language \\
\hline Delayed word recall & $\begin{array}{l}\text { Participants are instructed to write down the } \\
\text { words that were presented to them at the } \\
\text { beginning of the assessment. In this case, } 60 \mathrm{~s} \\
\text { were given to complete the task }\end{array}$ & $\begin{array}{l}\text { Number correct and } \\
\text { number of errors }\end{array}$ & Episodic memory \\
\hline
\end{tabular}


Table 2. Cont.

\begin{tabular}{|c|c|c|c|}
\hline Task & Descriptor & Scoring & Domain \\
\hline $\begin{array}{l}\text { Rapid visual information } \\
\text { processing }\end{array}$ & $\begin{array}{l}\text { A continuous series of single digits are } \\
\text { presented in the centre of the screen at the rate } \\
\text { of } 100 \text { per minute. Participants are required to } \\
\text { make a response when three consecutive odd } \\
\text { or three consecutive even digits are displayed. } \\
\text { In this case, the task lasted for } 5 \text { min, with eight } \\
\text { correct target strings presented in each minute. }\end{array}$ & $\begin{array}{l}\text { Accuracy }(\%) \text {, reaction } \\
\text { time for the correct } \\
\text { responses }(\mathrm{ms}) \text { and false } \\
\text { alarms (number) }\end{array}$ & Attention \\
\hline $\begin{array}{l}\text { Delayed word } \\
\text { recognition }\end{array}$ & $\begin{array}{l}\text { All target words that were shown during Word } \\
\text { presentation plus an equal number of decoys } \\
\text { are displayed on the screen one at a time. } \\
\text { Participants indicate if they remember seeing } \\
\text { the word earlier or not. }\end{array}$ & $\begin{array}{l}\text { Accuracy }(\%) \text { and } \\
\text { reaction time for the } \\
\text { correct responses (ms) }\end{array}$ & Episodic memory \\
\hline $\begin{array}{l}\text { Delayed picture } \\
\text { recognition }\end{array}$ & $\begin{array}{l}\text { All target pictures shown during Picture } \\
\text { presentation plus an equal number of decoys } \\
\text { are displayed on the screen one at a time. } \\
\text { Participants indicate if they remember seeing } \\
\text { the picture earlier or not. }\end{array}$ & $\begin{array}{l}\text { Accuracy }(\%) \text { and } \\
\text { reaction time for the } \\
\text { correct responses (ms) }\end{array}$ & Episodic memory \\
\hline
\end{tabular}

\subsection{Caffeine Research Visual Analogue Scales}

Prior to cognitive assessment, subjective state was assessed with the Caffeine Research Visual Analogue Scales [38], which have previously been used in caffeine research [16,21,39]. The following descriptors are presented on-screen: 'relaxed', 'alert', 'jittery', 'tired', 'tense', 'headache', 'overall mood', and 'mentally fatigued'. Participants are asked to rate how much these descriptors match their current state by placing an ' $x$ ' on a line with the end points labelled 'not at all' (left hand end) and 'extremely' (right hand end); with the exception of 'headache', which is labelled 'no headache' and 'extreme headache'; and 'overall mood', which is labelled 'very bad' and 'very good'. Ratings are scored as \% along the line from left to right.

\subsection{Computerised Location Learning—Learning Phase}

Location learning was assessed with a computerised task modified from Kessels et al. [40]. Participants are shown a grid containing pictures of objects. Following a timed delay they are shown an empty grid and asked to relocate the objects to the correct location shown to them previously. In the current study, this was repeated five times during the learning phase, with objects presented for $15 \mathrm{~s}$, a gap of $10 \mathrm{~s}$ before the empty grid was shown, and a pause of $5 \mathrm{~s}$ between each trial. For each of the five learning trials, a displacement score is calculated as the sum of the errors made for each object (calculated by counting the number of cells the object had to be moved both horizontally and vertically in order to be in the correct location) from each trial. A learning index is also calculated as the average relative difference in performance between trials $[((\mathrm{A}-\mathrm{B}) / \mathrm{A}+(\mathrm{B}-\mathrm{C}) / \mathrm{B}+(\mathrm{C}-\mathrm{D}) / \mathrm{C}+$ $(\mathrm{D}-\mathrm{E}) / \mathrm{D}) / 4]$.

\subsection{Computerised Location Learning-Delayed Trial}

During the delayed trial, which took place $30 \mathrm{~min}$ after completion of the learning phase, participants are again asked to place the objects in the correct location on the empty grid with no further prompting. The delayed trial is scored for displacement and delayed recall, which is calculated as the difference between displacement score on the final learning trial and the delayed trial.

\subsection{Driving Ability}

A PC based driving simulation (Driving Simulator 2013, Excalibur Publishing Limited, Banbury Oxfordshire, UK) was used to assess driving ability. Driving was controlled via a steering wheel and pedals with gears set to fully automatic. The task lasted for $3 \mathrm{~min}$ and is scored on the basis of adhering to road rules and driving ability. Specifically, the task is scored for errors, which are given when 
deviating too much from the track; deviating too much from the instructed directions; not indicating; speeding; colliding. If the drive ended (either because of collision or because of exceeding 10 errors) the task was restarted but no more than two restarts (three drives in total) were allowed.

\subsection{Procedure}

Potential participants attended the Brain Performance and Nutrition Research Centre at Northumbria University for an initial screening session where they gave informed consent prior to participation. Their eligibility was assessed in accordance with the criteria outlined in the 'Participants' section and training on the computerised tasks was provided. This consisted of five completions of cognitive tasks and took place on a single day. Participants attended three study visits, separated by at least seven days to allow for washout and to prevent confound due to caffeine abstention instructions. These instructions required abstention from caffeine from noon the day before study visits but this did not exceed $24 \mathrm{~h}$ in order to minimise any potential withdrawal effects. Consumption of alcohol and over-the-counter medication was also restricted for $24 \mathrm{~h}$ ( $48 \mathrm{~h}$ in the case of systemic antihistamines). On the morning of study visits, participants ate their usual breakfast at least $1 \mathrm{~h}$ prior to arrival at the laboratory with the time and composition of breakfast standardised across visits. Participants attended the laboratory at 9:45 a.m. and were screened to ensure eligibility for testing that day, this included checking they were in good health and had adhered to instructions regarding breakfast consumption, and caffeine, alcohol, and medication restrictions. A food diary was used to aid with breakfast standardisation and a saliva sample was obtained to confirm adherence to caffeine abstention instructions. All testing took place in a suite of dedicated temperature-controlled university laboratories with participants visually isolated from each other and wearing noise-reduction headphones to decrease the impact of any auditory distractions. Baseline assessments of cognition and mood were completed and participants were then given their drink for that day. After 30 min of rest in the laboratory, the learning phase of a computerised Location Learning Test (cLLT) was completed before parallel versions of the tasks completed at baseline. This was followed by the delayed trial of the cLLT, a driving simulation task and a final saliva sample for assessment of caffeine levels. At the end of the final visit only, participants were asked to guess which drink they believed they had consumed that day. See Figure 2 for a schematic depicting the study visit running order.

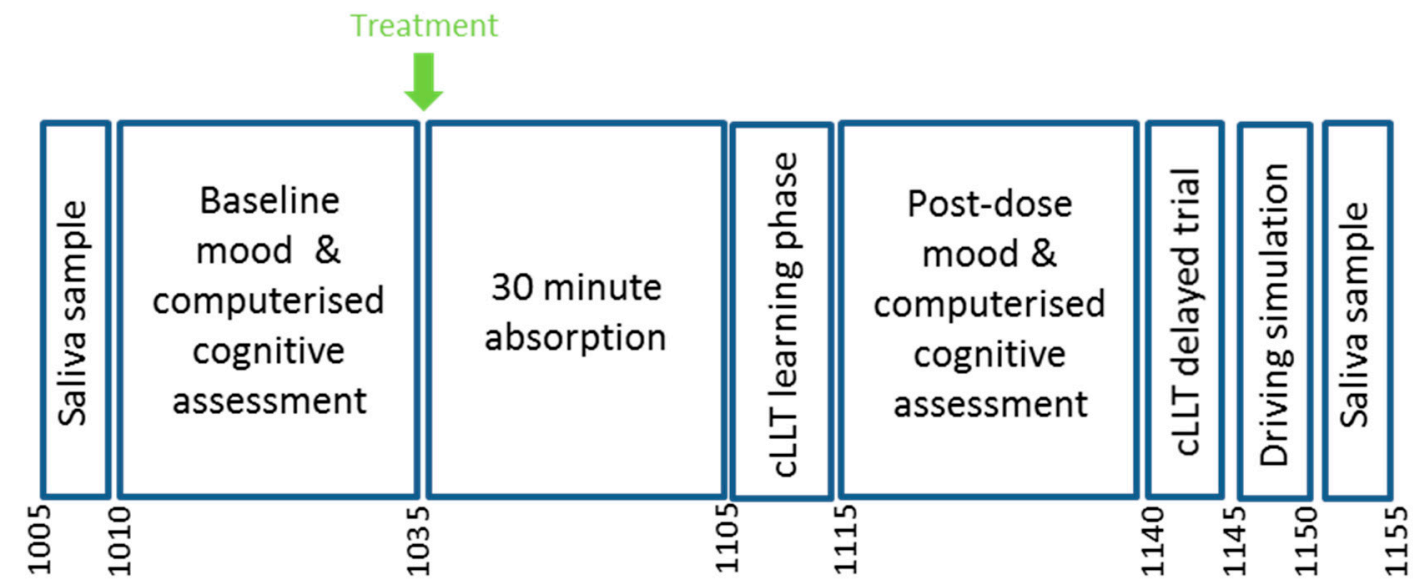

Figure 2. Study visit timeline.

\subsection{Statistics}

The post-dose outcome measures were modelled using the MIXED procedure in SPSS (version 24.0, IBM Corp., Armonk, NY, USA) which included the respective baseline values and the terms treatment, age, sex, treatment $\times$ age, treatment $\times$ sex and treatment $\times$ age $\times$ sex as fixed factors. In the case of computerised location learning and driving simulation, baseline values were taken from the final training session. Significant effects were followed up with Bonferroni corrected pairwise comparisons. 


\section{Results}

\subsection{Treatment-Related Effects}

\subsubsection{Salivary Caffeine}

Baseline salivary caffeine values were $0.17 \mu \mathrm{g} / \mathrm{mL}$, confirming adherence to caffeine abstention instructions. A significant main effect of treatment was observed on post-dose salivary caffeine $(\mathrm{F}(2,101.1)=155.6, p<0.0001)$. Pairwise comparisons revealed significantly greater levels following caffeinated coffee compared to placebo $(p<0.0001)$ and decaffeinated coffee $(p<0.0001)$. See Figure 3.

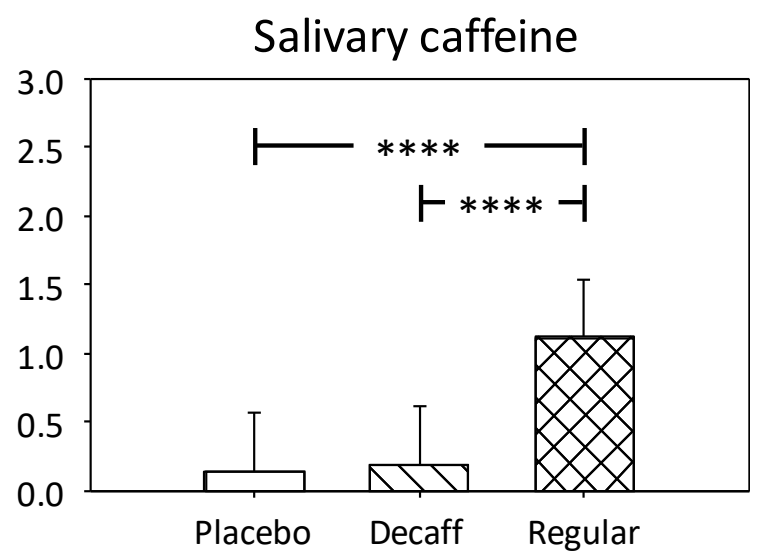

Figure 3. Adjusted means + standard error for salivary caffeine measured in $\mu \mathrm{g} / \mathrm{mL}$. Significant treatment effect ${ }^{* * * *} p<0.001$.

\subsubsection{Digit Vigilance}

A significant main effect of treatment was observed for digit vigilance accuracy $(\mathrm{F}(2,101.1)=4.44$, $p=0.014)$. Pairwise comparisons revealed significantly greater accuracy following regular coffee compared to decaffeinated coffee $(p=0.01)$. See Figure 4 a.

Digit vigilance reaction time was also significantly affected by treatment $(F(2,71.3)=5.07$, $p=0.009)$. Pairwise comparisons revealed significantly faster responses following regular coffee compared to placebo $(p=0.009)$. See Figure $4 b$.

a. Digit vigilance accuracy

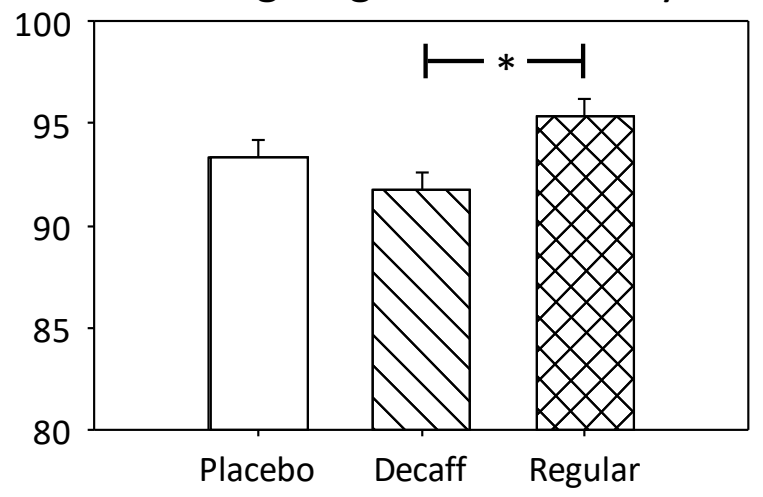

b. Digit vigilance reaction time

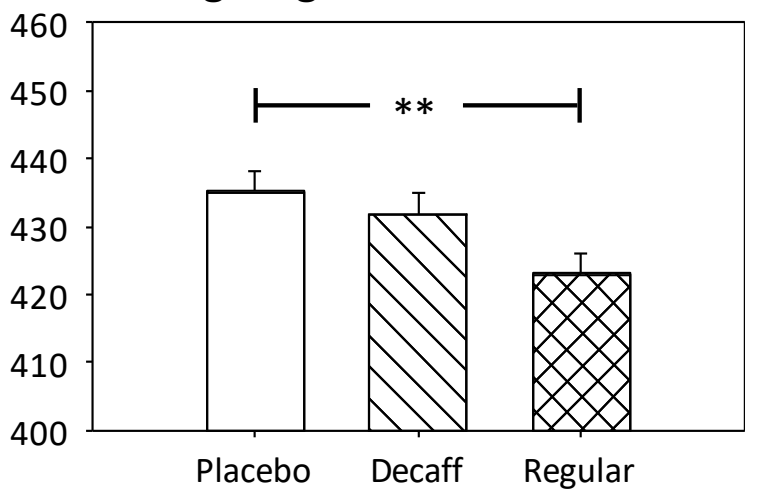

Figure 4. Cont. 


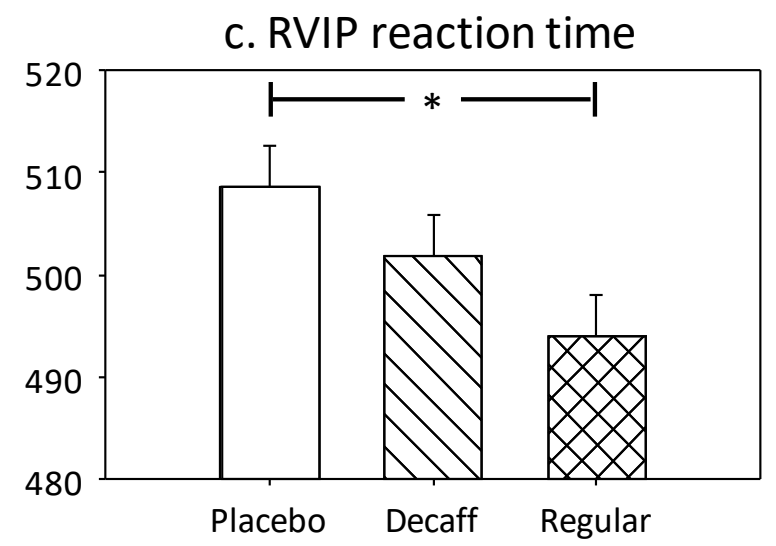

Figure 4. Adjusted means + standard error for those cognitive measures showing significant effects of treatment. (a) Digit vigilance accuracy; (b) Digit vigilance reaction time; (c) Rapid Visual Information Processing (RVIP) reaction time. Accuracy is measured as $\%$ and reaction time in milliseconds. ${ }^{*} p<0.05$; ** $p<0.01$.

\subsubsection{Rapid Visual Information Processing}

Rapid visual information processing reaction time showed a significant effect of treatment $(\mathrm{F}(2,102.9)=3.77, p=0.026)$. This was due to significantly faster responses following regular coffee compared to placebo $(p=0.02)$. See Figure $4 \mathrm{c}$. A significant treatment $\mathrm{x}$ sex interaction was also observed for false alarms $(\mathrm{F}(2,93.3)=4.55, p=0.013)$ but pairwise comparisons revealed no significant effects.

\subsubsection{Computerised Location Learning Delayed Trial}

Computerised location learning recall showed a significant treatment $\mathrm{x}$ sex interaction $(F(2,104)=3.46, p=0.035)$. However, pairwise comparisons revealed no significant effects.

\subsubsection{Alert}

Ratings of 'alertness' were significantly affected by treatment $(\mathrm{F}(2,106)=9.86, p<0.0001)$. This was due to significantly higher ratings following regular coffee $(p<0.0005)$ and decaffeinated coffee $(p=0.0048)$ compared to placebo. See Figure 5a.

\subsubsection{Tired}

A significant main effect of treatment on 'tired' ratings was also observed $(\mathrm{F}(2,101.4)=12.31$, $p=0.0001$ ). Pairwise comparisons revealed this was due to significantly lower ratings following regular coffee compared to decaffeinated coffee $(p=0.003)$ and placebo $(p<0.0001)$. See Figure $5 b$.

\subsubsection{Headache}

A significant main effect of treatment on headache ratings $(\mathrm{F}(2,92.9)=6.31, p=0.003)$ was due to significantly lower ratings following regular coffee compared to decaffeinated coffee $(p=0.0049)$ and placebo $(p=0.015)$. See Figure $5 c$.

\subsubsection{Overall Mood}

'Overall mood' was significantly affected by treatment $(F(2,105.8)=5.56, p<0.005)$. This was due to significantly higher ratings following regular coffee compared to placebo $(p=0.004)$. See Figure $5 \mathrm{~d}$. 


\subsubsection{Mental Fatigue}

A significant main effect of treatment on 'mental fatigue' ratings was also observed $(\mathrm{F}(2,97.5)=4.43, p=0.014)$. Pairwise comparisons revealed this was due to significantly lower ratings following regular coffee compared to placebo $(p=0.01)$. See Figure 5 e.

\subsubsection{Jittery}

A significant treatment $\times$ age $\times$ sex interaction was observed on jittery ratings $(F(3,76.2)=3.01$, $p=0.035)$. Pairwise comparisons revealed significantly higher ratings following regular coffee compared to placebo in young females $(p=0.046)$ and compared to decaffeinated coffee in older males (0.045). See Figure $5 f$.

Unadjusted means, standard deviations, and $F$ and $p$ values for all factors (treatment, age, sex) and their interactions can be found in Tables S1-S3.

a. Alert

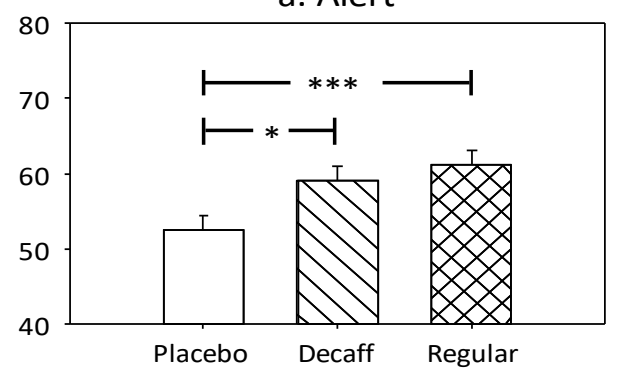

c. Headache

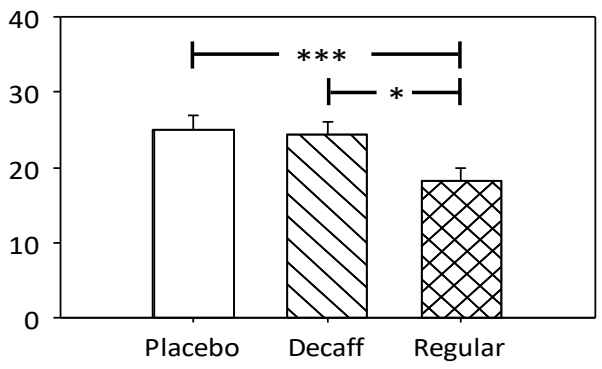

e. Mental fatigue

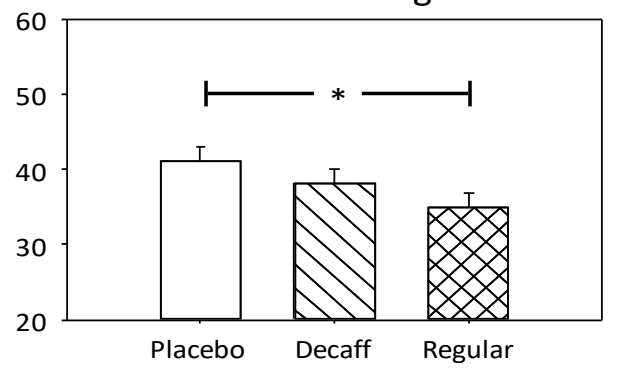

b. Tired

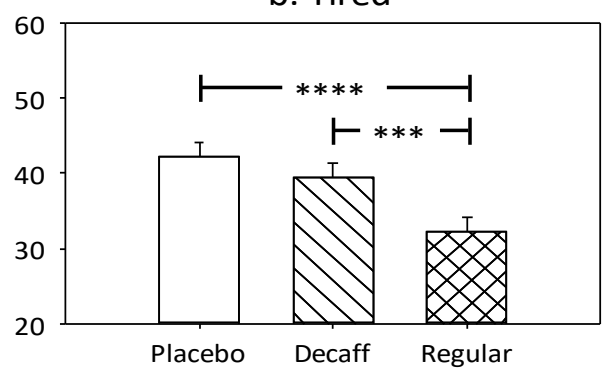

d. Overall mood

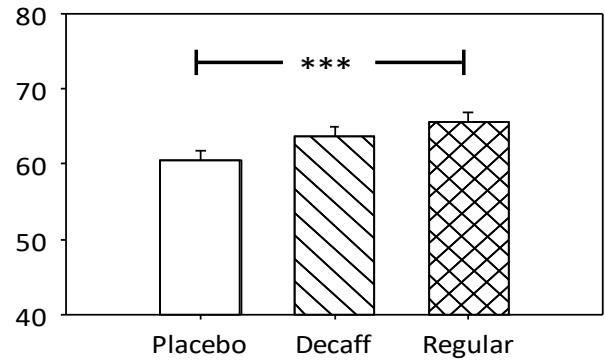

f. Jittery

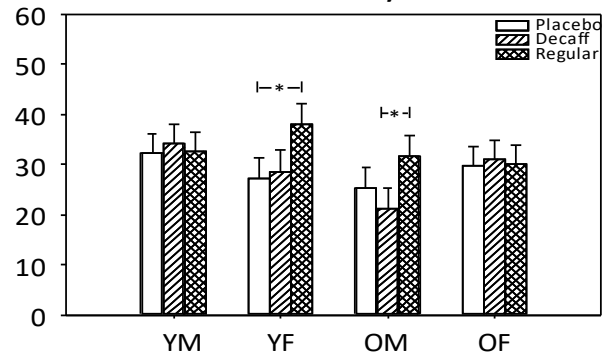

Figure 5. Adjusted means + standard error for those mood measures showing significant treatment-related effects. (a) Alert; (b) Tired; (c) Headache; (d) Overall mood; (e) Mental fatigue; (f) Jittery. Ratings are measured as \% along a visual analogue scale with higher values indicating greater response. $\mathrm{YM}=$ young male; $\mathrm{YF}=$ young female; $\mathrm{OM}=$ older male; $\mathrm{OF}=$ older female; * $p<0.05$; *** $p<0.005 ; * * * * 0.001$. 


\subsection{Treatment Guess}

Seventy-one percent of participants correctly guessed which drink they had received at the final visit. Eighty-one percent correctly guessed they had received regular coffee, $72 \%$ correctly identified decaffeinated coffee, and $58 \%$ were able to correctly identify placebo as their final drink.

\section{Discussion}

Consumption of $220 \mathrm{~mL}$ of regular coffee containing $100 \mathrm{mg}$ caffeine led to faster responses during digit vigilance and rapid visual information processing tasks when compared to placebo, and to increased digit vigilance accuracy when compared to decaffeinated coffee. In terms of mood effects, ratings of alertness and overall mood were higher and mental fatigue ratings lower following regular coffee compared to placebo. Tiredness and headache ratings were lower following regular coffee compared to placebo and decaffeinated coffee. Rating of jitteriness was the only outcome to show an interaction with sex and age indicating higher ratings following regular coffee when compared to placebo in young females and when compared to decaffeinated coffee in older males. Decaffeinated coffee also engendered an increase in subjective alertness, compared to placebo, whereas accuracy of digit vigilance and tired and headache ratings were impaired in comparison to regular coffee. A beneficial effect of decaffeinated coffee was also observed following a treatment $\times$ age $\times$ sex interaction, which indicated that ratings of jitteriness were significantly lower following decaffeinated compared to regular coffee in older men. The pattern of response to decaffeinated coffee generally fell between responses to regular coffee and placebo. Specifically, numeric working memory accuracy and reaction time, reaction time for attention tasks and mood ratings, all followed the order of placebo > decaffeinated > regular coffee (see Supplementary Tables), with the exception of relaxed and tense ratings which showed a preferential effect of decaffeinated coffee.

The findings with regards regular coffee are largely in line with the reported effects of caffeine, which only has a consistent beneficial effect on attention task performance and subjective alertness/arousal [16-18]. Whilst this could be taken as support for the notion that caffeine is the sole contributor to the effects, the finding of psychoactive effects of decaffeinated coffee, in terms of increased alertness when compared to placebo and a pattern of lower effects than regular coffee in comparison to placebo, supports the suggestion of a modulatory role for the non-caffeine compounds within coffee. The effects of decaffeinated coffee presented here are broadly in line with previous results showing impairment to accuracy of a sustained attention task in comparison to regular coffee [25] and increases in alertness when compared to placebo and the phenolic acid CGA in isolation [26]. Previous studies have highlighted CGA as a potentially important component of coffee. However, whilst there is some evidence for beneficial modulation of coffee's effects by increasing CGA content [25], the effects of CGA in isolation were largely negative [26]. This potentially highlights an issue in applying a reductionist approach to nutritional interventions where complex interactions between many different components may be required to see optimum results. Although composition is varied depending on roasting and brewing techniques, caffeine generally only accounts for $\sim 1 \%$ and CGA $10 \%$ of the weight of coffee beans, this leaves almost $90 \%$ of the constituents unaccounted for. It is also important to note that the CGA profile may be altered as part of the decaffeination process and therefore any analysis of effects must take account of the impact of decaffeination on other constituents [41].

The observed benefits for regular coffee are expected due to the known effects of caffeine in antagonising adenosine $\mathrm{A}_{1}$ and $\mathrm{A}_{2 \mathrm{~A}}$ receptors thereby, increasing oxygen metabolism [42] and upregulating various neurotransmitters including noradrenaline, dopamine, serotonin, acetylcholine, glutamate, and GABA [15]. Caffeine and its metabolites also have a number of mechanistic properties that make them liable to have a modulatory or interactive effect when caffeine is co-consumed with other bioactive compounds. These include the inhibition of enzymes involved in the breakdown of neurotransmitters (e.g., acetylcholinesterase and monoamine oxidase) and cellular signalling molecules (e.g., phosphodiesterase and PARP (poly(ADP-ribose)polymerase)) $[43,44]$ and a role as a competitive 
substrate for a number of cytochrome P450 (CYP) enzymes (CYP2A1, CYP2E1, and CYP1A1) that metabolise endogenous and exogenous chemicals in the human body [45-47]. Of particular relevance here, low-doses of caffeine have therefore been shown to increase the bioavailability of phenolic compounds [48-50] and have a synergistic effect in terms of the cardiovascular benefits of polyphenols.

Coffee also has the potential to impact glucose metabolism as evidenced by an increase in insulin sensitivity observed following decaffeinated coffee when compared to placebo [51]. Interestingly, this effect was not apparent following regular coffee, which may be due to counteractive effects of caffeine and non-caffeine components within regular coffee. Support for this comes from data showing decreased insulin sensitivity following caffeine [52]. Moreover, area under the curve (AUC) profiles for serum insulin indicate that caffeine increases AUC when compared to decaffeinated coffee and placebo, whilst regular coffee produced a trend towards the same when compared to decaffeinated coffee, with similar profiles evinced for glucose AUC [53]. CGA derivatives have been shown to increase insulin sensitivity in rats [54], and further support for the role of phenolic compounds in this effect comes from data showing modulation of glucose and insulin response following phenolic-rich berries [55-57] as well as a reduction in the postprandial blood glucose response following grape seed extract [58]. Similarly, caffeine is known to have a vasoconstrictive effect, including reduced cerebral blood flow (CBF) [39], whereas phenolic-rich foods have demonstrated the opposite effect. Of particular relevance is the ability of phenolic-rich cocoa to increase CBF when compared to a phenolic-poor control matched for methylxanthine content $[59,60]$. These findings indicate the ability of coffee components to counteract the negative effects of caffeine and a potential synergy whereby phenolic compounds increase $\mathrm{CBF}$, and therefore oxygen supply, whilst caffeine increases brain activity and subsequent oxygen metabolism. It is also possible that caffeine increases absorption of phenolics as has been shown following consumption of cocoa [48] but is as yet untested following coffee.

A further consideration is that due to a focus on psychoactive effects of caffeine, the cognitive and mood effects of coffee have typically been measured at 30 to 120 min post-dose coinciding with a peak in caffeine levels at around 40-min post-ingestion [61]. However, analysis of the fate of CGA following coffee consumption shows that whilst a number of phenolic acids and their derivatives peak between 30 and $60 \mathrm{~min}$, others do not appear until between 4- and 6-h post-ingestion [62]. It is therefore necessary to extend the testing period in order to fully examine the impact of these metabolites. This is also true in relation to caffeine, which has a half-life of around $5 \mathrm{~h} \mathrm{[63],} \mathrm{and} \mathrm{has}$ demonstrated behavioural effects up to $8 \mathrm{~h}$ post-dose [64]. It is therefore probable that any effects of coffee observed at 6-h would represent an interaction between phenolic acids and caffeine and the measurement of biomarkers would aid in elucidating the role of each.

Learning and episodic memory tasks showed the expected effect in terms of significantly lower performance in the older cohort when compared to young (see Supplementary Tables). However, no interactions between age and treatment were observed on any cognitive measure. Whilst learning and memory are not typically susceptible to caffeine, it has been proposed that these tasks may show sensitivity in low arousal situations as is expected in the elderly as energetic resources diminish [65]. However, in the current study there was no evidence of higher arousal in the young sample when compared to the older participants on subjective measures of 'arousal' or psychomotor tasks. This may suggest that the older cohort studied here were relatively high functioning, as is supported by their status being higher than national averages both in relation to fruit and vegetable consumption and education level [66]. It has also been suggested that cognitive benefits of caffeine consumption may be more pronounced in those aged over 80 years [31,67]. Therefore, the findings reported here do not preclude interaction effects in an older sample with poorer nutritional status and/or lower education level.

Similarly, although sex differences were observed in the current study, these did not interact with treatment as may have been expected from data showing greater benefits of coffee consumption in women than men [30]. However, the potential mechanisms underlying sex differences following habitual consumption, including sex steroid levels [68,69], haemodynamic mechanisms [70], and uric 
acid responses [71,72], are unlikely to exert effects over a 30-min time period. Furthermore, given the impact of the menstrual cycle and hormonal contraception on metabolism, it is possible that any differential sex effects in the younger cohort were obscured by the lack of control for menstrual cycle phase and the inclusion of four hormonal contraceptive users in this study. This also potentially explains large variations in salivary caffeine following regular coffee in young females that were not observed in the young male group. Similar large variations in response were shown for older men and women indicating individual differences in response to caffeine. This variability is largely due to differences in CYP1A2 activity, which is influenced by a number of factors including sex and genetic polymorphisms [73].

Polymorphisms of the ADORA2A gene may only explain the age, sex, treatment interaction, which indicated that older men and younger women experienced greater feelings of jitteriness following regular coffee. It has previously been reported that those who are $\mathrm{T} / \mathrm{T}$ homozygous at nucleotide positions 1976C > T and 2592Tins experience increases in anxiety after caffeine administration that are not observed in the other genotypic groups $[74,75]$. Moreover, sex differences in response have been noted for 1976TT homozygotes, whereby females are more susceptible to anxiogenic effects of caffeine than males [76,77]. Interestingly, whilst there is evidence for reduced caffeine intake in 1976TT homozygotes [78], others have shown that intake of coffee, but not other sources of caffeine, is increased. It was also shown that increased habitual consumption moderated anxiogenic effects of caffeine such that they were only observed in non/light consumers of caffeine, irrespective of genotype [79]. This indicates that even in those with a genetic predisposition, tolerance to anxiogenic effects can occur with habitual consumption. As the older men in the current study consumed less coffee than their female counterparts, this may in part explain the specificity of 'jittery' effects observed here. However, there are a multitude of factors that impact on interindividual differences [80], which require further exploration before definitive conclusions can be reached.

In the current study, although the addition of a true placebo builds on research previously limited to comparing effects following caffeinated and decaffeinated coffee, one important limitation is the omission of a caffeine-only arm. The inclusion of a caffeine-only condition would have allowed a direct comparison of any synergistic effects between caffeine and the other bioactive compounds in coffee, including the phenolic compounds. It may also have facilitated in blinding of drinks. Although the placebo drink in the current study was somewhat effective in that $42 \%$ incorrectly identified it, only 1 of 19 participants mistook it for regular coffee. It is important to note that this measure was only included at the end of the final visit when all three drinks had been consumed and, therefore, does not rule out the blinding of participants at earlier visits. However, as the stimulant effects of caffeine are easily detected, the inclusion of a caffeine-containing 'placebo' would provide an active control for regular coffee and reduce the ability of participants to correctly identify regular coffee.

The findings presented here suggest behavioural activity of coffee beyond its caffeine content. In fact, only one cognitive measure and two subjective measures showed significant differences between regular and decaffeinated coffee in favour of regular coffee. This highlights two key issues with studies which compare regular and decaffeinated coffee. Firstly, these studies attribute any differential effects to caffeine without considering the potential for interaction with other components. Secondly, any synergistic effects of caffeine and other coffee components within regular coffee are likely to be underestimated due to the potential for behavioural effects of decaffeinated coffee used as the control. If the effects of regular coffee are to be fully understood, it is important that future research compares these to the equivalent dose of caffeine, decaffeinated coffee, and placebo. Furthermore, research in this area must include plasma levels of potentially important compounds, including phenolic compounds. This would allow assessment of the impact of caffeine on the pharmacodynamic profile of other components in coffee. An extended testing period is also recommended in order to capture effects of colonic metabolites of phenolics appearing at $\sim 8 \mathrm{~h}$. Further research is also required in which cognition is measured alongside potential underlying mechanisms including, but not limited to, glucoregulation and modulation of cerebral haemodynamics. Finally, in order to capture the impact 
of interindividual differences in metabolism of caffeine and other components of coffee on behavioural outcomes, genetic factors should also be considered.

Supplementary Materials: The following are available online at http:/ /www.mdpi.com/2072-6643/10/10/1386/ s1, Table S1: saliva; Table S2: cognition; Table S3: mood.

Author Contributions: C.F.H-R., P.A.J., J.S.F., F.L.D. and D.O.K. contributed to the design of the study; J.S.F. and F.L.D. collected the data; S.L.B. conducted the salivary analysis; C.F.H.-R. conducted the data analysis; all authors contributed to and reviewed the final publication.

Funding: This research and APC were funded by the Institute for Scientific Information on Coffee (ISIC) who collaborated on aspects of the design but had no further involvement in the study.

Conflicts of Interest: The authors declare no conflicts of interest.

\section{References}

1. Rodriguez-Artalejo, F.; Lopez-Garcia, E. Coffee consumption and cardiovascular disease: A condensed review of epidemiological evidence and mechanisms. J. Agric. Food Chem. 2018, 66, 5257-5263. [CrossRef] [PubMed]

2. Jiang, X.B.; Zhang, D.F.; Jiang, W.J. Coffee and caffeine intake and incidence of type 2 diabetes mellitus: A meta-analysis of prospective studies. Eur. J. Nutr. 2014, 53, 25-38. [CrossRef] [PubMed]

3. Andersen, L.F.; Jacobs, D.R., Jr.; Carlsen, M.H.; Blomhoff, R. Consumption of coffee is associated with reduced risk of death attributed to inflammatory and cardiovascular diseases in the Iowa women's health study. Am. J. Clin. Nutr. 2006, 83, 1039-1046. [CrossRef] [PubMed]

4. Greenberg, J.A.; Dunbar, C.C.; Schnoll, R.; Kokolis, R.; Kokolis, S.; Kassotis, J. Caffeinated beverage intake and the risk of heart disease mortality in the elderly: A prospective analysis. Am. J. Clin. Nutr. 2007, 85, 392-398. [CrossRef] [PubMed]

5. Freedman, N.D.; Park, Y.; Abnet, C.C.; Hollenbeck, A.R.; Sinha, R. Association of coffee drinking with total and cause-specific mortality. N. Engl. J. Med. 2012, 366, 1891-1904. [CrossRef] [PubMed]

6. Gunter, M.J.; Murphy, N.; Cross, A.J.; Dossus, L.; Dartois, L.; Fagherazzi, G.; Kaaks, R.; Kuhn, T.; Boeing, H.; Aleksandrova, K.; et al. Coffee drinking and mortality in 10 european countries: A multinational cohort study. Ann. Intern. Med. 2017, 167, 236-247. [CrossRef] [PubMed]

7. Park, S.Y.; Freedman, N.D.; Haiman, C.A.; Le Marchand, L.; Wilkens, L.R.; Setiawan, V.W. Association of coffee consumption with total and cause-specific mortality among nonwhite populations. Ann. Intern. Med. 2017, 167, 228-235. [CrossRef] [PubMed]

8. Jarvis, M.J. Does caffeine intake enhance absolute levels of cognitive performance? Psychopharmacology 1993, 110, 45-52. [CrossRef] [PubMed]

9. Araujo, L.F.; Giatti, L.; Reis, R.C.; Goulart, A.C.; Schmidt, M.I.; Duncan, B.B.; Ikram, M.A.; Barreto, S.M. Inconsistency of association between coffee consumption and cognitive function in adults and elderly in a cross-sectional study (elsa-brasil). Nutrients 2015, 7, 9590-9601. [CrossRef] [PubMed]

10. Eskelinen, M.H.; Kivipelto, M. Caffeine as a protective factor in dementia and Alzheimer's disease. J. Alzheimers Dis. 2010, 20, S167-S174. [CrossRef] [PubMed]

11. Eskelinen, M.H.; Ngandu, T.; Tuomilehto, J.; Soininen, H.; Kivipelto, M. Midlife coffee and tea drinking and the risk of late-life dementia: A population-based caide study. J. Alzheimers Dis. 2009, 16, 85-91. [CrossRef] [PubMed]

12. Maia, L.; de Mendonca, A. Does caffeine intake protect from alzheimer's disease? Eur. J. Neurol. 2002, 9, 377-382. [CrossRef] [PubMed]

13. Lindsay, J.; Laurin, D.; Verreault, R.; Hebert, R.; Helliwell, B.; Hill, G.B.; McDowell, I. Risk factors for alzheimer's disease: A prospective analysis from the canadian study of health and aging. Am. J. Epidemiol. 2002, 156, 445-453. [CrossRef] [PubMed]

14. Liu, Q.P.; Wu, Y.F.; Cheng, H.Y.; Xia, T.; Ding, H.; Wang, H.; Wang, Z.M.; Xu, Y. Habitual coffee consumption and risk of cognitive decline/dementia: A systematic review and meta-analysis of prospective cohort studies. Nutrition 2016, 32, 628-636. [CrossRef] [PubMed]

15. Fredholm, B.B.; Battig, K.; Holmen, J.; Nehlig, A.; Zvartau, E.E. Actions of caffeine in the brain with special reference to factors that contribute to its widespread use. Pharmacol. Rev. 1999, 51, 83-133. [PubMed] 
16. Haskell, C.F.; Kennedy, D.O.; Wesnes, K.A.; Scholey, A.B. Cognitive and mood improvements of caffeine in habitual consumers and habitual non-consumers of caffeine. Psychopharmacology 2005, 179, 813-825. [CrossRef] [PubMed]

17. Smit, H.J.; Rogers, P.J. Effects of low doses of caffeine on cognitive performance, mood and thirst in low and higher caffeine consumers. Psychopharmacology 2000, 152, 167-173. [CrossRef] [PubMed]

18. Childs, E.; de Wit, H. Subjective, behavioral, and physiological effects of acute caffeine in light, nondependent caffeine users. Psychopharmacology 2006, 185, 514-523. [CrossRef] [PubMed]

19. Renouf, M.; Marmet, C.; Giuffrida, F.; Lepage, M.; Barron, D.; Beaumont, M.; Williamson, G.; Dionisi, F. Dose-response plasma appearance of coffee chlorogenic and phenolic acids in adults. Mol. Nutr. Food Res. 2014, 58, 301-309. [CrossRef] [PubMed]

20. Dodd, F.L.; Kennedy, D.O.; Riby, L.M.; Haskell-Ramsay, C.F. A double-blind, placebo-controlled study evaluating the effects of caffeine and l-theanine both alone and in combination on cerebral blood flow, cognition and mood. Psychopharmacology 2015, 232, 2563-2576. [CrossRef] [PubMed]

21. Haskell, C.F.; Kennedy, D.O.; Milne, A.L.; Wesnes, K.A.; Scholey, A.B. The effects of l-theanine, caffeine and their combination on cognition and mood. Biol. Psychol. 2008, 77, 113-122. [CrossRef] [PubMed]

22. Giles, G.E.; Mahoney, C.R.; Brunye, T.T.; Taylor, H.A.; Kanarek, R.B. Caffeine and theanine exert opposite effects on attention under emotional arousal. Can. J. Physiol. Pharmacol. 2017, 95, 93-100. [CrossRef] [PubMed]

23. Andrews, S.E.; Blumenthal, T.D.; Flaten, M.A. Effects of caffeine and caffeine-associated stimuli on the human startle eyeblink reflex. Pharmacol. Biochem. Behav. 1998, 59, 39-44. [CrossRef]

24. Ferruzzi, M.G. The influence of beverage composition on delivery of phenolic compounds from coffee and tea. Physiol. Behav. 2010, 100, 33-41. [CrossRef] [PubMed]

25. Cropley, V.; Croft, R.; Silber, B.; Neale, C.; Scholey, A.; Stough, C.; Schmitt, J. Does coffee enriched with chlorogenic acids improve mood and cognition after acute administration in healthy elderly? A pilot study. Psychopharmacology 2012, 219, 737-749. [CrossRef] [PubMed]

26. Camfield, D.A.; Silber, B.Y.; Scholey, A.B.; Nolidin, K.; Goh, A.; Stough, C. A randomised placebo-controlled trial to differentiate the acute cognitive and mood effects of chlorogenic acid from decaffeinated coffee. PLoS ONE 2013, 8, e82897. [CrossRef] [PubMed]

27. Duff, S.J.; Hampson, E. A sex difference on a novel spatial working memory task in humans. Brain Cognit. 2001, 47, 470-493. [CrossRef] [PubMed]

28. Herlitz, A.; Rehnman, J. Sex differences in episodic memory. Curr. Dir. Psychol. Sci. 2008, 17, 52-56. [CrossRef]

29. van Gelder, B.M.; Buijsse, B.; Tijhuis, M.; Kalmijn, S.; Giampaoli, S.; Nissinen, A.; Kromhout, D. Coffee consumption is inversely associated with cognitive decline in elderly european men: The fine study. Eur. J. Clin. Nutr. 2007, 61, 226-232. [CrossRef] [PubMed]

30. Arab, L.; Biggs, M.L.; O'Meara, E.S.; Longstreth, W.T.; Crane, P.K.; Fitzpatrick, A.L. Gender differences in tea, coffee, and cognitive decline in the elderly: The cardiovascular health study. J. Alzheimers Dis. 2011, 27, 553-566. [CrossRef] [PubMed]

31. Ritchie, K.; Carriere, I.; de Mendonca, A.; Portet, F.; Dartigues, J.F.; Rouaud, O.; Barberger-Gateau, P.; Ancelin, M.L. The neuroprotective effects of caffeine: A prospective population study (the three city study). Neurology 2007, 69, 536-545. [CrossRef] [PubMed]

32. Henry, C.J.; Lightowler, H.J.; Marchini, J. Intra-individual variation in resting metabolic rate during the menstrual cycle. Br. J. Nutr. 2003, 89, 811-817. [CrossRef] [PubMed]

33. Lane, J.D.; Steege, J.F.; Rupp, S.L.; Kuhn, C.M. Menstrual cycle effects on caffeine elimination in the human female. Eur. J. Clin. Pharmacol. 1992, 43, 543-546. [CrossRef] [PubMed]

34. James, J.E.; Rogers, P.J. Effects of caffeine on performance and mood: Withdrawal reversal is the most plausible explanation. Psychopharmacology 2005, 182, 1-8. [CrossRef] [PubMed]

35. Smith, A.P.; Christopher, G.; Sutherland, D. Acute effects of caffeine on attention: A comparison of non-consumers and withdrawn consumers. J. Psychopharmacol. 2013, 27, 77-83. [CrossRef] [PubMed]

36. Kennedy, D.O.; Wightman, E.L.; Reay, J.L.; Lietz, G.; Okello, E.J.; Wilde, A.; Haskell, C.F. Effects of resveratrol on cerebral blood flow variables and cognitive performance in humans: A double-blind, placebo-controlled, crossover investigation. Am. J. Clin. Nutr. 2010, 91, 1590-1597. [CrossRef] [PubMed] 
37. Stonehouse, W.; Conlon, C.A.; Podd, J.; Hill, S.R.; Minihane, A.M.; Haskell, C.; Kennedy, D. Dha supplementation improved both memory and reaction time in healthy young adults: A randomized controlled trial. Am. J. Clin. Nutr. 2013, 97, 1134-1143. [CrossRef] [PubMed]

38. Rogers, P.J.; Martin, J.; Smith, C.; Heatherley, S.V.; Smit, H.J. Absence of reinforcing, mood and psychomotor performance effects of caffeine in habitual non-consumers of caffeine. Psychopharmacology 2003, 167, 54-62. [CrossRef] [PubMed]

39. Kennedy, D.O.; Haskell, C.F. Cerebral blood flow and behavioural effects of caffeine in habitual and non-habitual consumers of caffeine: A near infrared spectroscopy study. Biol. Psychol. 2011, 86, 298-306. [CrossRef] [PubMed]

40. Kessels, R.P.; Nys, G.M.; Brands, A.M.; van den Berg, E.; Van Zandvoort, M.J. The modified location learning test: Norms for the assessment of spatial memory function in neuropsychological patients. Arch. Clin. Neuropsychol. 2006, 21, 841-846. [CrossRef] [PubMed]

41. Fujioka, K.; Shibamoto, T. Chlorogenic acid and caffeine contents in various commercial brewed coffees. Food Chem. 2008, 106, 217-221. [CrossRef]

42. Griffeth, V.E.; Perthen, J.E.; Buxton, R.B. Prospects for quantitative fmri: Investigating the effects of caffeine on baseline oxygen metabolism and the response to a visual stimulus in humans. Neuroimage 2011, 57, 809-816. [CrossRef] [PubMed]

43. Geraets, L.; Moonen, H.J.; Wouters, E.F.; Bast, A.; Hageman, G.J. Caffeine metabolites are inhibitors of the nuclear enzyme poly(adp-ribose)polymerase-1 at physiological concentrations. Biochem. Pharmacol. 2006, 72, 902-910. [CrossRef] [PubMed]

44. Zulli, A.; Smith, R.M.; Kubatka, P.; Novak, J.; Uehara, Y.; Loftus, H.; Qaradakhi, T.; Pohanka, M.; Kobyliak, N.; Zagatina, A.; et al. Caffeine and cardiovascular diseases: Critical review of current research. Eur. J. Nutr. 2016, 55, 1331-1343. [CrossRef] [PubMed]

45. Carrillo, J.A.; Benitez, J. Clinically significant pharmacokinetic interactions between dietary caffeine and medications. Clin. Pharmacokinet. 2000, 39, 127-153. [CrossRef] [PubMed]

46. Gunes, A.; Dahl, M.L. Variation in cyp1a2 activity and its clinical implications: Influence of environmental factors and genetic polymorphisms. Pharmacogenomics 2008, 9, 625-637. [CrossRef] [PubMed]

47. Gambaro, S.E.; Moretti, R.; Tiribelli, C.; Gazzin, S. Brain cytochrome p450 enzymes: A possible therapeutic targets for neurological diseases. Ther. Targets Neurol. Dis. 2015, 2, e598.

48. Sansone, R.; Ottaviani, J.I.; Rodriguez-Mateos, A.; Heinen, Y.; Noske, D.; Spencer, J.P.; Crozier, A.; Merx, M.W.; Kelm, M.; Schroeter, H.; et al. Methylxanthines enhance the effects of cocoa flavanols on cardiovascular function: Randomized, double-masked controlled studies. Am. J. Clin. Nutr. 2017, 105, 352-360. [CrossRef] [PubMed]

49. Dulloo, A.G.; Duret, C.; Rohrer, D.; Girardier, L.; Mensi, N.; Fathi, M.; Chantre, P.; Vandermander, J. Efficacy of a green tea extract rich in catechin polyphenols and caffeine in increasing 24-h energy expenditure and fat oxidation in humans. Am. J. Clin. Nutr. 1999, 70, 1040-1045. [CrossRef] [PubMed]

50. Nakagawa, K.; Nakayama, K.; Nakamura, M.; Sookwong, P.; Tsuduki, T.; Niino, H.; Kimura, F.; Miyazawa, T. Effects of co-administration of tea epigallocatechin-3-gallate (EGCG) and caffeine on absorption and metabolism of EGCG in humans. Biosci. Biotechnol. Biochem. 2009, 73, 2014-2017. [CrossRef] [PubMed]

51. Reis, C.E.G.; Paiva, C.; Amato, A.A.; Lofrano-Porto, A.; Wassell, S.; Bluck, L.J.C.; Dorea, J.G.; da Costa, T.H.M. Decaffeinated coffee improves insulin sensitivity in healthy men. Br. J. Nutr. 2018, 119, 1029-1038. [CrossRef] [PubMed]

52. MacKenzie, T.; Comi, R.; Sluss, P.; Keisari, R.; Manwar, S.; Kim, J.; Larson, R.; Baron, J.A. Metabolic and hormonal effects of caffeine: Randomized, double-blind, placebo-controlled crossover trial. Metabolism 2007, 56, 1694-1698. [CrossRef] [PubMed]

53. Battram, D.S.; Arthur, R.; Weekes, A.; Graham, T.E. The glucose intolerance induced by caffeinated coffee ingestion is less pronounced than that due to alkaloid caffeine in men. J. Nutr. 2006, 136, 1276-1280. [CrossRef] [PubMed]

54. Adisakwattana, S.; Moonsan, P.; Yibchok-Anun, S. Insulin-releasing properties of a series of cinnamic acid derivatives in vitro and in vivo. J. Agric. Food Chem. 2008, 56, 7838-7844. [CrossRef] [PubMed]

55. Torronen, R.; Kolehmainen, M.; Sarkkinen, E.; Mykkanen, H.; Niskanen, L. Postprandial glucose, insulin, and free fatty acid responses to sucrose consumed with blackcurrants and lingonberries in healthy women. Am. J. Clin. Nutr. 2012, 96, 527-533. [CrossRef] [PubMed] 
56. Torronen, R.; Kolehmainen, M.; Sarkkinen, E.; Poutanen, K.; Mykkanen, H.; Niskanen, L. Berries reduce postprandial insulin responses to wheat and rye breads in healthy women. J. Nutr. 2013, 143, 430-436. [CrossRef] [PubMed]

57. Torronen, R.; Sarkkinen, E.; Niskanen, T.; Tapola, N.; Kilpi, K.; Niskanen, L. Postprandial glucose, insulin and glucagon-like peptide 1 responses to sucrose ingested with berries in healthy subjects. Br. J. Nutr. 2012, 107, 1445-1451. [CrossRef] [PubMed]

58. Sapwarobol, S.; Adisakwattana, S.; Changpeng, S.; Ratanawachirin, W.; Tanruttanawong, K.; Boonyarit, W. Postprandial blood glucose response to grape seed extract in healthy participants: A pilot study. Pharmacogn. Mag. 2012, 8, 192-196. [CrossRef] [PubMed]

59. Francis, S.T.; Head, K.; Morris, P.G.; Macdonald, I.A. The effect of flavanol-rich cocoa on the fMRI response to a cognitive task in healthy young people. J. Cardiovasc. Pharmacol. 2006, 47, S215-S220. [CrossRef] [PubMed]

60. Lamport, D.J.; Pal, D.; Moutsiana, C.; Field, D.T.; Williams, C.M.; Spencer, J.P.; Butler, L.T. The effect of flavanol-rich cocoa on cerebral perfusion in healthy older adults during conscious resting state: A placebo controlled, crossover, acute trial. Psychopharmacology 2015, 232, 3227-3234. [CrossRef] [PubMed]

61. Liguori, A.; Hughes, J.R.; Grass, J.A. Absorption and subjective effects of caffeine from coffee, cola and capsules. Pharmacol. Biochem. Behav. 1997, 58, 721-726. [CrossRef]

62. Stalmach, A.; Williamson, G.; Crozier, A. Impact of dose on the bioavailability of coffee chlorogenic acids in humans. Food Funct. 2014, 5, 1727-1737. [CrossRef] [PubMed]

63. Blanchard, J.; Sawers, S.J. Comparative pharmacokinetics of caffeine in young and elderly men. J. Pharmacokinet. Biopharm. 1983, 11, 109-126. [CrossRef] [PubMed]

64. Haskell-Ramsay, C.F.; Jackson, P.A.; Forster, J.S.; Robertson, B.C.; Kennedy, D.O. Acute effects of three doses of caffeine on attention, motor speed and mood over an 8-hour period. Appetite 2018, in press. [CrossRef]

65. van Boxtel, M.; Schmitt, J. Coffee, Tea, Chocolate, and the Brain, 1st ed.; Nehlig, A., Ed.; CRC Press: Boca Raton, FL, USA, 2004.

66. Office for National Statistics (ONS). 2011 Census: Key Statistics for England and Wales. March 2011. Available online: http:/ / www.ons.gov.uk/ons/dcp171778_290685.pdf (accessed on 28 September 2018).

67. Johnson-Kozlow, M.; Kritz-Silverstein, D.; Barrett-Connor, E.; Morton, D. Coffee consumption and cognitive function among older adults. Am. J. Epidemiol. 2002, 156, 842-850. [CrossRef] [PubMed]

68. Ascherio, A.; Weisskopf, M.G.; O’Reilly, E.J.; McCullough, M.L.; Calle, E.E.; Rodriguez, C.; Thun, M.J. Coffee consumption, gender, and parkinson's disease mortality in the cancer prevention study II cohort: The modifying effects of estrogen. Am. J. Epidemiol. 2004, 160, 977-984. [CrossRef] [PubMed]

69. Ferrini, R.L.; Barrett-Connor, E. Caffeine intake and endogenous sex steroid levels in postmenopausal women. The rancho bernardo study. Am. J. Epidemiol. 1996, 144, 642-644. [CrossRef] [PubMed]

70. Hartley, T.R.; Lovallo, W.R.; Whitsett, T.L. Cardiovascular effects of caffeine in men and women. Am. J. Cardiol. 2004, 93, 1022-1026. [CrossRef] [PubMed]

71. Kiyohara, C.; Kono, S.; Honjo, S.; Todoroki, I.; Sakurai, Y.; Nishiwaki, M.; Hamada, H.; Nishikawa, H.; Koga, H.; Ogawa, S.; et al. Inverse association between coffee drinking and serum uric acid concentrations in middle-aged japanese males. Br. J. Nutr. 1999, 82, 125-130. [PubMed]

72. Perna, L.; Mons, U.; Schottker, B.; Brenner, H. Association of cognitive function and serum uric acid: Are cardiovascular diseases a mediator among women? Exp. Gerontol. 2016, 81, 37-41. [CrossRef] [PubMed]

73. Rasmussen, B.B.; Brix, T.H.; Kyvik, K.O.; Brosen, K. The interindividual differences in the 3-demthylation of caffeine alias cyp1a2 is determined by both genetic and environmental factors. Pharmacogenetics 2002, 12, 473-478. [CrossRef] [PubMed]

74. Alsene, K.; Deckert, J.; Sand, P.; de Wit, H. Association between A2a receptor gene polymorphisms and caffeine-induced anxiety. Neuropsychopharmacology 2003, 28, 1694-1702. [CrossRef] [PubMed]

75. Childs, E.; Hohoff, C.; Deckert, J.; Xu, K.; Badner, J.; de Wit, H. Association between adora2a and drd2 polymorphisms and caffeine-induced anxiety. Neuropsychopharmacology 2008, 33, 2791-2800. [CrossRef] [PubMed]

76. Gajewska, A.; Blumenthal, T.D.; Winter, B.; Herrmann, M.J.; Conzelmann, A.; Muhlberger, A.; Warrings, B.; Jacob, C.; Arolt, V.; Reif, A.; et al. Effects of ADORA2A gene variation and caffeine on prepulse inhibition: A multi-level risk model of anxiety. Prog. Neuropsychopharmacol. Biol. Psychiatry 2013, 40, 115-121. [CrossRef] [PubMed] 
77. Domschke, K.; Gajewska, A.; Winter, B.; Herrmann, M.J.; Warrings, B.; Muhlberger, A.; Wosnitza, K.; Glotzbach, E.; Conzelmann, A.; Dlugos, A.; et al. ADORA2A gene variation, caffeine, and emotional processing: A multi-level interaction on startle reflex. Neuropsychopharmacology 2012, 37, 759-769. [CrossRef] [PubMed]

78. Cornelis, M.C.; El-Sohemy, A.; Campos, H. Genetic polymorphism of the adenosine A2A receptor is associated with habitual caffeine consumption. Am. J. Clin. Nutr. 2007, 86, 240-244. [CrossRef] [PubMed]

79. Rogers, P.J.; Hohoff, C.; Heatherley, S.V.; Mullings, E.L.; Maxfield, P.J.; Evershed, R.P.; Deckert, J.; Nutt, D.J. Association of the anxiogenic and alerting effects of caffeine with ADORA2A and adora1 polymorphisms and habitual level of caffeine consumption. Neuropsychopharmacology 2010, 35, 1973-1983. [CrossRef] [PubMed]

80. Nehlig, A. Interindividual differences in caffeine metabolism and factors driving caffeine consumption. Pharmacol. Rev. 2018, 70, 384-411. [CrossRef] [PubMed]

(C) 2018 by the authors. Licensee MDPI, Basel, Switzerland. This article is an open access article distributed under the terms and conditions of the Creative Commons Attribution (CC BY) license (http://creativecommons.org/licenses/by/4.0/). 